INPLASY

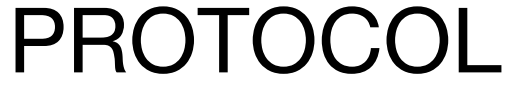

To cite: Lu et al. Effect of massage on myofascial pain syndrome. Inplasy protocol 2021120088. doi:

10.37766/inplasy2021.12.0088

Received: 20 December 2021

Published: 20 December 2021

Corresponding author:

Xingang Lu

11231280003@fudan.edu.com

Author Affiliation:

Fudan University

Support: Long Hua project (Y2006).

Review Stage at time of this submission: Data analysis Completed but not published.

Conflicts of interest:

None declared.

\section{Effect of massage on myofascial pain syndrome}

\author{
$L u, X^{1} ; L u, W^{2}$.
}

Review question / Objective: Massage is one of complementary and alternative medicine (CAM) therapies. Massage can relieve pain in many disorders, for example, fibromyalgia. However, many treatments and systematic review overlapping fibromyalgia and Myofascial pain. The systematic review and meta analysis focus on myofascial pain is lack up to date. This systematic review and meta analysis focus on myofascial pain aims to investigate the effect of massage on myofascial pain.

Condition being studied: Pain is defined as unpleasant sensory and emotional experiences that are associated with actual or potential tissue damage. Myofascial pain is a prevalent chronic musculoskeletal pain condition. The prevalence of Myofascial pain varies from $30 \%$ to as high as $90 \%$ of patient worldwide. Myofascial pain is characterized by the presence of myofascial trigger points. Myofascial pain syndrome is a musculoskeletal condition that is thought to stem from localized. Myofascial pain characterized as dull, aching, boring, and burning. Massage was used in many RCTs for therapies of mypfascial pain.

INPLASY registration number: This protocol was registered with the International Platform of Registered Systematic Review and Meta-Analysis Protocols (INPLASY) on 20 December 2021 and was last updated on 20 December 2021 (registration number INPLASY2021120088).

\section{INTRODUCTION}

Review question / Objective: Massage is one of complementary and alternative medicine (CAM) therapies. Massage can relieve pain in many disorders, for example, fibromyalgia. However, many treatments and systematic review overlapping fibromyalgia and Myofascial pain. The systematic review and meta analysis focus on myofascial pain is lack up to date. This systematic review and meta analysis focus 
on myofascial pain aims to investigate the effect of massage on myofascial pain.

Condition being studied: Pain is defined as unpleasant sensory and emotional experiences that are associated with actual or potential tissue damage. Myofascial pain is a prevalent chronic musculoskeletal pain condition. The prevalence of Myofascial pain varies from $30 \%$ to as high as $90 \%$ of patient worldwide. Myofascial pain is characterized by the presence of myofascial trigger points. Myofascial pain syndrome is a musculoskeletal condition that is thought to stem from localized. Myofascial pain characterized as dull, aching, boring, and burning. Massage was used in many RCTs for therapies of mypfascial pain.

\section{METHODS}

Search strategy: Language was limited to English. Databases will be retrieved from the earliest data available to 2021/12/1. The search strategies are composed of such items: ("massage") AND ("myofascial pain syndrome") AND ("randomized controlled trial"). The references of previous literatures will be fully tracked. The http:// www.clinicaltrial.gov was searched for potential studies which is conducting or planed.

Participant or population: Subjects with myofascial pain syndrome.

Intervention: Massage.

Comparator: sham control or normal care.

Study designs to be included: RCTs.

Eligibility criteria: (1) Randomized controlled trials. (2) The trails contained the subjects with clinical diagnosis of myofascial pain syndrome syndrome. (4) The controls were sham control normal care.

Information sources: These electronic databases will be researched: Pubmed, the Cochrane Library, Exceerpta Medica
Database (EMBASE), Web of Science, Physiotherapy evidence database.

Main outcome(s): Pain index such as VAS or numerical rate pain scales.

Additional outcome(s): Quality of life; mood; anxiety.

Quality assessment / Risk of bias analysis: Two review authors will independently assess the risk of bias in included studies according to the criteria in the Cochrane Handbook for Systematic Reviews of Interventions as follows: the random sequence generation, allocation concealment, incomplete outcome data, blinding (participants, personnel, and outcome assessment), selective reporting, and other biases. After that, authors independently judged each study as having bias, a high risk of bias, or an unclear risk of bias.

Strategy of data synthesis: Review Manager (Revman, Version 5.3) software provided by the Cochrane Collaboration was used for data analysis. For continuous variables, standardized mean difference (SMD) and 95\% confidence interval (Cl) were used for statistics. The heterogeneity tests of each outcome were performed using Chi-squared test and 12 statistic. When no significant heterogeneity was observed $(12<50 \%)$, the fixed-effects model was used to perform meta-analysis. When heterogeneity was detected $(P<0.05$ and $\mathrm{I} 2 \geq 50 \%$ ), a random-effects model was used.

Subgroup analysis: If available, subgroup analyses will be performed following below items in our plan: type of massage (spinal manipulation OR musculoskeletal manipulation OR tuina OR Shiatsu OR Acupressure OR Reflexology OR Manual Lymphatic Drainage OR connective tissue massae OR myofascial realease), male/ female of subjects, location of study, duration of treatment.

Sensitivity analysis: The sensitivity analyses were also planned to explore possible reasons for statistical 
heterogeneity when $12>50 \%$. The methods containing removing one by one, regression, etc.

Language: English.

Country(ies) involved: P.R.China.

Keywords: massage; myofascial pain; trigger point; vas.

Contributions of each author:

Author 1 - Xingang Lu.

Email: 11231280003@fudan.edu.cn

Author 2 - Wei Lu.

Email: luwei@shutem.edu.cn 